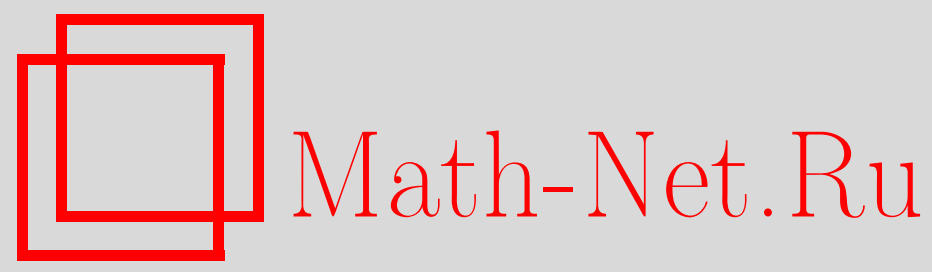

М. А. Башов, Минимизация двусторонней тени в единичном кубе, Дискрет. матем., 2011, том 23, выпуск 4, 115-132

DOI: https://doi.org/10.4213/dm1165

Использование Общероссийского математического портала Math-Net.Ru подразумевает, что вы прочитали и согласны с пользовательским соглашением http://www . mathnet.ru/rus/agreement

Параметры загрузки:

IP: 54.162 .27 .143

26 апреля 2023 г., 17:41:25 
УДК 519.1

\title{
Минимизация двусторонней тени в единичном кубе
}

\author{
(c) 2011 г. M. А. Башов
}

\begin{abstract}
Рассматривается задача минимизации двусторонней тени подмножества слоя единичного куба. Показано, что пересечение шара Хэмминга радиуса 2 со слоем куба имеет минимальную двустороннюю тень, и описаны минимальные по двусторонней тени множества меньшей мощности.
\end{abstract}

\section{1. Введение}

Известная изопериметрическая задача на плоскости заключается в нахождении простой замкнутой кривой фиксированной длины, ограничивающей наибольшую площадь. Одним из аналогов этой задачи в дискретных пространствах, таких, как единичный куб, является задача о поиске подмножества слоя фиксированной мощности с минимальной тенью. Задача с односторонней тенью возникает, например, при установлении асимптотики числа монотонных булевых функций [1].

Пусть $\mathscr{A}$ - подмножество слоя $B_{k}^{n}$ единичного куба $B^{n}$. Тенью вниз $\triangle \mathscr{A}$ называется множество таких наборов $\tilde{\alpha}$ из слоя $B_{k-1}^{n}$, что существует набор $\tilde{\beta} \in \mathscr{A}, \tilde{\beta}>\tilde{\alpha}$. Аналогично, тенью вверх $\nabla \mathscr{A}$ называется множество таких наборов $\tilde{\alpha}$ из слоя $B_{k+1}^{n}$, что существует набор $\tilde{\beta} \in \mathscr{A}, \tilde{\beta}<\tilde{\alpha}$. Двусторонней тенью $\triangle \mathscr{A}$ называется объединение множеств $\triangle \mathscr{A}$ и $\nabla \mathscr{A}$.

Множество $\mathscr{A} \subseteq B_{k}^{n}$ называется минимальным по тени вниз (тени вверх, двусторонней тени), если $|\triangle \mathscr{A}| \leqslant|\triangle \mathscr{B}|$ (соответственно, $|\nabla \mathscr{A}| \leqslant|\nabla \mathscr{B}|,|\nabla \mathscr{A}| \leqslant|\nabla \mathscr{B}|)$ для любого $\mathscr{B} \subseteq B_{k}^{n}$, такого, что $|\mathscr{B}|=|\mathscr{A}|$.

Краскал [2] и Катона [3] установили, что начальный лексикографический отрезок слоя единичного куба минимален по тени вниз. Мерс [4] и Фюреди и Григгс [5] доказали, что лексикографический отрезок является единственным с точностью до изоморфизма минимальным множеством тогда и только тогда, когда он является замкнутым множеством, то есть не существует такой точки вне отрезка, что ее тень вниз целиком лежала бы в тени вниз отрезка.

Клементс и Линдстрем [6] обобщили результат Краскала и Катоны на произвольное декартово произведение цепей. Аналоги теоремы Краскала-Катоны доказаны также для декартова произведения звезд [7] и двойственного частично упорядоченного множества [8].

В данной работе рассмотрена задача минимизации двусторонней тени подмножеств слоя $B_{k}^{n}$, и описаны минимальные множества мощности, не превосходящей $1+k(n-k)$. 


\section{2. Основные понятия}

Пусть $\tilde{\alpha} \in B_{k}^{n}$. Кругом $\mathscr{b}_{r}(\tilde{\alpha})$ назовем множество $\left\{\tilde{\beta} \in B_{k}^{n} \mid \rho(\tilde{\alpha}, \tilde{\beta}) \leqslant 2 r\right\}$, то есть пересечение шара Хэмминга радиуса $2 r$ и слоя $B_{k}^{n}$. Заметим, что

$$
\begin{aligned}
\left|\mathscr{b}_{r}(\tilde{\alpha})\right| & =\sum_{i=0}^{r}\left(\begin{array}{l}
k \\
i
\end{array}\right)\left(\begin{array}{c}
n-k \\
i
\end{array}\right), \\
\left|\Delta \mathscr{C}_{r}(\tilde{\alpha})\right| & =\sum_{i=0}^{r}\left(\begin{array}{c}
k \\
i+1
\end{array}\right)\left(\begin{array}{c}
n-k \\
i
\end{array}\right), \\
\left|\nabla \mathscr{C}_{r}(\tilde{\alpha})\right| & =\sum_{i=0}^{r}\left(\begin{array}{l}
k \\
i
\end{array}\right)\left(\begin{array}{l}
n-k \\
i+1
\end{array}\right) .
\end{aligned}
$$

В частности, если $n=2 N, k=N$, то

$$
\begin{aligned}
\left|\mathscr{C}_{r}(\tilde{\alpha})\right| & =\sum_{i=0}^{r}\left(\begin{array}{c}
N \\
i
\end{array}\right)^{2}, \\
\left|\nabla \mathscr{C}_{r}(\tilde{\alpha})\right| & =2 \sum_{i=0}^{r}\left(\begin{array}{c}
N \\
i+1
\end{array}\right)\left(\begin{array}{c}
N \\
i
\end{array}\right),
\end{aligned}
$$

И

$$
\frac{\left|\Delta \mathscr{C}_{N / 2}(\tilde{\alpha})\right|}{\left|\mathscr{C}_{N / 2}(\tilde{\alpha})\right|} \sim \frac{\left|\nabla \mathscr{C}_{N / 2}(\tilde{\alpha})\right|}{\left|\mathscr{C}_{N / 2}(\tilde{\alpha})\right|} \sim 1 .
$$

Таким образом, множества $\mathscr{C}_{N / 2}(\tilde{\alpha})$ асимптотически минимальны по тени вниз и по тени вверх, а значит, и по двусторонней тени. Естественно предположить, что круги $\mathfrak{b}_{r}(\tilde{\alpha})$ являются минимальными по двусторонней тени множествами. В этой работе данная гипотеза доказывается для $r=1$.

При поиске минимальных по двусторонней тени множеств удобнее перейти от слоя булева куба $B_{k}^{n}$ к семейству $\left(\begin{array}{c}{[n]} \\ k\end{array}\right) k$-элементных подмножеств $[n]=\{1,2, \ldots, n\}$. Для этого набору $\tilde{\alpha} \in B_{k}^{n}$ сопоставим множество $A$ чисел $i$ таких, что $\alpha_{n-i+1}=1$. Подмножествам $B_{k}^{n}$ соответствуют семейства множеств из $\left(\begin{array}{c}{[n]} \\ k\end{array}\right)$, и определения теней, минимальных множеств и лексикографического порядка переносятся на семейства.

Будем обозначать символами $A, B, C, D$ элементы $\left(\begin{array}{c}{[n]} \\ k\end{array}\right)$, под $a_{i}$ будем понимать элемент $A$, причем будем считать, что $a_{i}$ упорядочены по возрастанию, то есть $A=\left\{a_{1}, a_{2}, \ldots, a_{k}\right\}$ и $a_{1}<a_{2}<\ldots<a_{k}$.

Введем на $\left(\begin{array}{c}n] \\ k\end{array}\right)$ отношение частичного порядка. Пусть $A$ и $B$ - элементы $\left(\begin{array}{c}{[n]} \\ k\end{array}\right)$. Положим $A \sqsubseteq B$, если $a_{i} \leqslant b_{i}$ для $i=1, \ldots, k$. На рис. 1 изображены диаграммы Хассе для частично упорядоченных множеств $\left(\left(\begin{array}{c}{[4]} \\ 2\end{array}\right), \sqsubseteq\right),\left(\left(\begin{array}{c}{[6]} \\ 3\end{array}\right), \sqsubseteq\right)$ и $\left(\left(\begin{array}{c}{[7]} \\ 3\end{array}\right), \sqsubseteq\right)$.

Семейство $\mathscr{F} \subseteq\left(\begin{array}{c}{[n]} \\ k\end{array}\right)$ называется идеалом $\mathcal{M}(n, k)=\left(\left(\begin{array}{c}{[n]} \\ k\end{array}\right), \sqsubseteq\right)$, если из того, что $A \in \mathscr{F}$ следует, что любое множество $B$ такое, что $B \sqsubseteq A$, входит в $\mathscr{F}$.

Обозначим через $\mathscr{C}_{1}(n, k)$ семейство, соответствующее кругу $\mathscr{C}_{1}(\underbrace{00 \ldots 0}_{n-k} \underbrace{11 \ldots 1}_{k})$. Заметим, что $\mathscr{b}_{1}(n, k)$ является идеалом $\mathcal{M}(n, k)$. На изображенных на рис. 2 диаграммах Хассе $\left(\begin{array}{c}{[5]} \\ 2\end{array}\right)$ и $\left(\begin{array}{c}{[6]} \\ 3\end{array}\right)$ выделены точки, принадлежащие соответственно $\mathscr{C}_{1}(5,2)$ и $\mathscr{C}_{1}(6,3)$. 


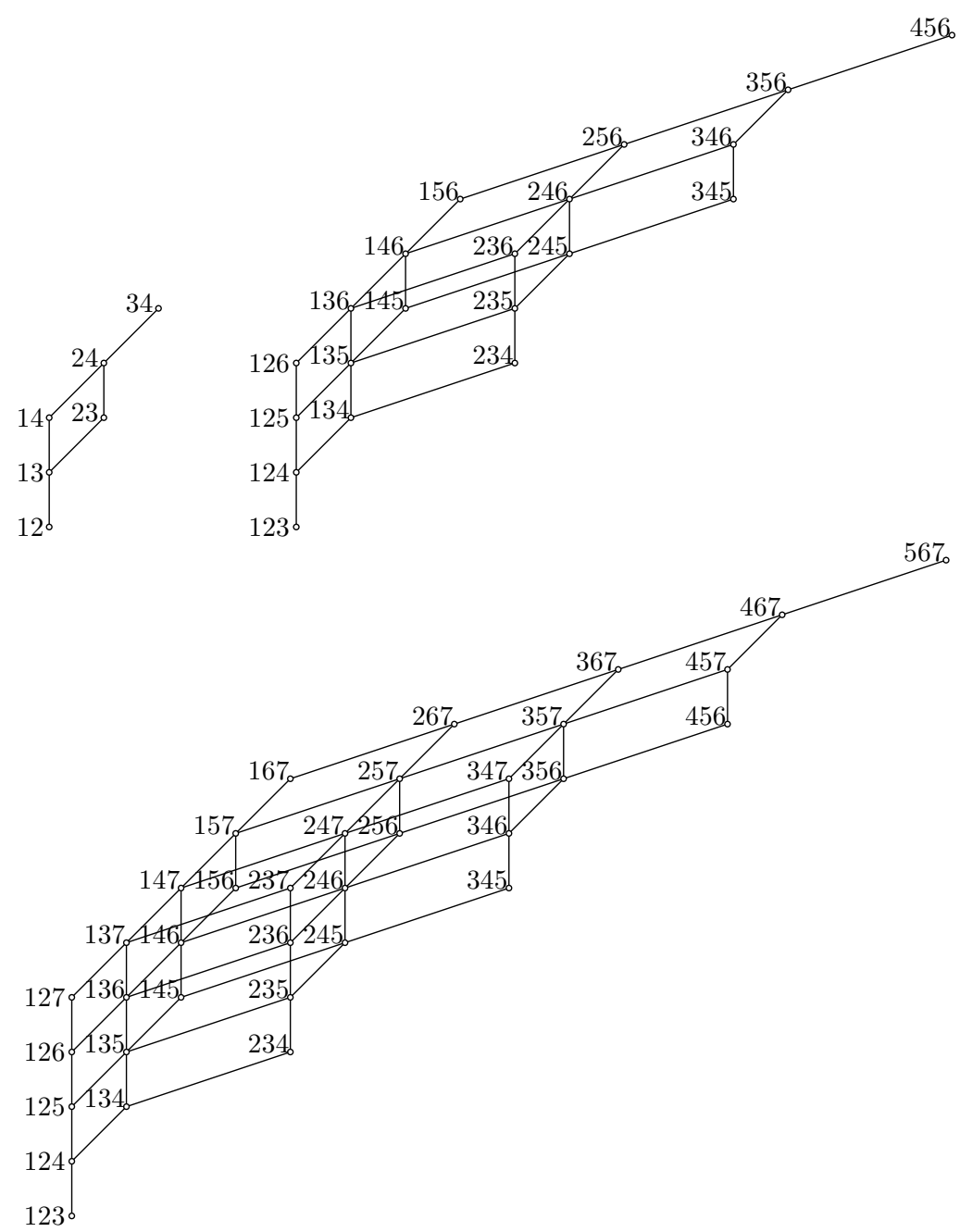

Рис. 1.

Пусть $A \in\left(\begin{array}{c}{[n]} \\ k\end{array}\right), \mathscr{F} \subseteq\left(\begin{array}{c}{[n]} \\ k\end{array}\right)$. Рассмотрим следующий оператор сдвига:

$$
S_{i j}(\mathscr{F}, A)= \begin{cases}A, & \text { если либо } j \notin A, \text { либо } i \in A, \\ A, & \text { если } A \cup\{i\} \backslash\{j\} \in \mathscr{F}, \\ A \cup\{i\} \backslash\{j\} & \text { в противном случае. }\end{cases}
$$

\section{Обозначим}

$$
S_{i j}(\mathscr{F})=\left\{S_{i j}(\mathscr{F}, A) \mid A \in \mathscr{F}\right\} .
$$

Семейство $\mathscr{F} \subseteq\left(\begin{array}{c}{[n]} \\ k\end{array}\right)$ называется несдвигаемым, если $S_{i j}(\mathscr{F})=\mathscr{F}$ для любых $i$ и $j$ таких, что $1 \leqslant i<j \leqslant n$. 

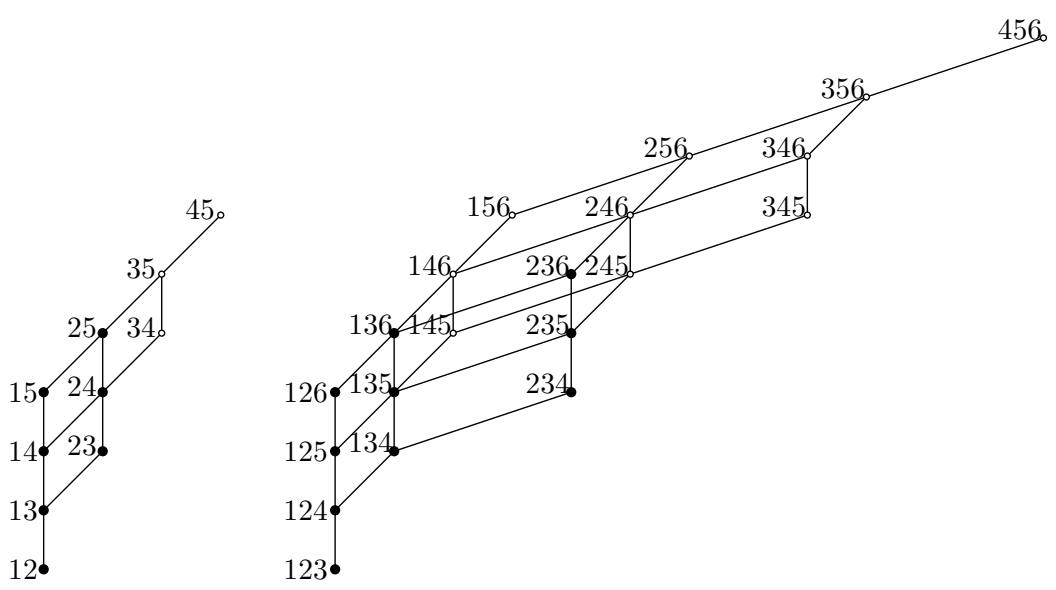

Рис. 2.

\section{3. Свойства оператора сдвига}

Предложение 1 ([9, 10]). Пусть $\mathscr{F} \subseteq\left(\begin{array}{c}{[n]} \\ k\end{array}\right)$. Тогда верны следующие утверждения:

(1) $\left|S_{i j}(\mathscr{F})\right|=|\mathscr{F}|$,

(2) $\left|\triangle S_{i j}(\mathscr{F})\right| \leqslant|\triangle \mathscr{F}|$,

(3) $\left|\nabla S_{i j}(\mathscr{F})\right| \leqslant|\nabla \mathscr{F}|$,

(4) существует последовательность операторов сдвига $S_{i_{1} j_{1}}, S_{i_{2} j_{2}}, \ldots, S_{i_{l} j_{l}}$ такая, что $S_{i_{l} j_{l}}\left(S_{i_{l-1} j_{l-1}}\left(\ldots S_{i_{1} j_{1}}(\mathscr{F}) \ldots\right)\right.$ является несдвигаемым семейством.

Итак, оператор сдвига не меняет мощность семейства, не увеличивает мощность тени, и любое семейство можно привести к несдвигаемому. Значит, для решения задачи минимизации достаточно рассматривать лишь несдвигаемые семейства.

Следующее утверждение дает критерий того, что оператор $S_{i j}$ строго уменьшает мощность тени $\mathscr{F}$.

Предложение 2. Неравенство $\left|\nabla S_{i j}(\mathscr{F})\right|<|\nabla \mathscr{F}|$ имеет место тогда и только тогда, когда для некоторого $A \in\left(\begin{array}{c}{[n] \backslash\{i, j\}} \\ k-2\end{array}\right)$ семейство F्F содержит элементы $\left\{i, m_{1}\right\} \cup A u$ $\left\{j, m_{2}\right\} \cup A$, причем либо $\{j\} \cup A \notin \Delta S_{i j}(\mathscr{F})$, либо $\left\{j, m_{1}, m_{2}\right\} \cup A \notin \nabla S_{i j}(\mathscr{F})$.

Доказательство. Разобьем семейство $\mathscr{F}$ на пять частей:

$$
\begin{aligned}
\mathscr{F} & =\mathscr{F}_{1} \cup \mathscr{F}_{2} \cup \mathscr{F}_{3} \cup \mathscr{F}_{4} \cup \mathscr{F}_{5}, \\
\mathscr{F}_{1} & =\{A \in \mathscr{F} \mid i \in A, j \in A\}, \\
\mathscr{F}_{2} & =\{A \in \mathscr{F} \mid i \notin A, j \notin A\}, \\
\mathscr{F}_{3} & =\{A \in \mathscr{F} \mid i \in A, A \cup\{j\} \backslash\{i\} \in \mathscr{F}\} \cup\left\{A \in \mathscr{F} \mid j \in A, A \cup\{i\} \backslash\{j\} \in \mathscr{F}_{3}\right\}, \\
\mathscr{F}_{4} & =\{A \in \mathscr{F} \mid i \in A, j \notin A\} \backslash \mathscr{F}_{3}, \\
\mathscr{F}_{5} & =\{A \in \mathscr{F} \mid i \notin A, j \in A\} \backslash \mathscr{F}_{3} .
\end{aligned}
$$


Положим

$$
\mathscr{F}_{0}=\mathscr{F} \backslash \mathscr{F}_{5} .
$$

Из определения оператора $S_{i j}$ следует, что

$$
S_{i j}\left(\mathscr{F}_{r}\right)=\mathscr{F}_{r}, \quad r \leqslant 4,
$$

откуда следует, что

$$
\begin{aligned}
\nabla_{i j}\left(\mathscr{F}_{r}\right) & =\nabla_{\mathscr{F}_{r}}, \\
S_{i j}\left(\mathscr{F}_{5}\right) & =\left\{A \cup\{i\} \backslash\{j\} \mid A \in \mathscr{F}_{5}\right\} .
\end{aligned}
$$

Таким образом,

$$
\begin{aligned}
\left|S_{i j}\left(\mathscr{F}_{5}\right)\right| & =\left|\mathscr{F}_{5}\right|, \\
\left|\nabla S_{i j}\left(\mathscr{F}_{5}\right)\right| & =\left|\nabla \mathscr{F}_{5}\right| .
\end{aligned}
$$

Поэтому $\left|\nabla S_{i j}(\mathscr{F})\right|<|\nabla \mathscr{F}|$ тогда и только тогда, когда

$$
\left|\nabla S_{i j}\left(\mathscr{F}_{5}\right) \cap \nabla \mathscr{F}_{0}\right|>\left|\nabla \mathscr{F}_{5} \cap \nabla \mathscr{F}_{0}\right|,
$$

то есть существует такой $A \in \mathscr{F}_{5}$, что

$$
\begin{array}{r}
\nabla A \cap \nabla \mathscr{F}_{0}=\varnothing, \\
\nabla S_{i j}(\mathscr{F}, A) \cap \nabla \mathscr{F}_{0} \neq \varnothing .
\end{array}
$$

Эти условия на $A$ эквивалентны тому, что существует такое $B \in \mathscr{F}_{0}$, что $i \in B, j \notin B$, $|A \cap B|=k-2$ и $\{\{j\} \cup(A \cap B),\{j\} \cup A \cup B\} \nsubseteq \triangle S_{i j}(\mathscr{F})$.

\section{4. Свойства частичного порядка $\sqsubseteq$}

Предложение 3. Семейство F्F несдвигаемо тогда и только тогда, когда $\mathscr{F}$ является идеалом $M(n, k)$.

Доказательство. Поскольку при $i<j$ для произвольного семейства $\mathscr{F} \subseteq\left(\begin{array}{c}{[n]} \\ k\end{array}\right)$ и произвольного множества $A \in\left(\begin{array}{c}{[n]} \\ k\end{array}\right)$ выполнено $S_{i j}(\mathscr{F}, A) \sqsubseteq A$, любой идеал $M(n, k)$ является несдвигаемым семейством.

Пусть $\mathscr{F}$ - несдвигаемое семейство. Предположим, что $\mathscr{F}-$ не идеал, то есть существуют такие $A$ и $B$, что $A \in \mathscr{F}, B \notin \mathscr{F}$ и $B \sqsubseteq A$. Не ограничивая общности, можно считать, что $B$ непосредственно предшествует $A$, то есть не существует такого $C$, отличного от $A$ и $B$, что $B \sqsubseteq C \sqsubseteq A$. Тогда из определения отношения $\sqsubseteq$ получаем, что $B=A \cup\{i\} \backslash\{i+1\}$ для некоторого $i$, и $S_{i j}(\mathscr{F}, A)=B$ при $j=i+1$, противоречие с несдвигаемостью $\mathscr{F}$.

Предложение 4. Пусть $A, B \in\left(\begin{array}{c}{[n]} \\ k\end{array}\right)$, и двусторонние тени $A$ и B имеют непустое пересечение. Тогда либо $A \sqsubseteq B$, либо $B \sqsubseteq A$.

Доказательство. Без ограничения общности можно считать, что $\triangle A \cap \Delta B \neq \varnothing$. Пусть $C \in \triangle A \cap \triangle B$. Тогда, по определению тени вниз,

$$
A=C \cup\{x\}, \quad B=C \cup\{y\} .
$$

Пусть $x \leqslant y$. Тогда по определению $A \sqsubseteq B$. 
Таким образом, если двусторонние тени двух множеств пересекаются, то эти множества сравнимы. Это позволяет установить формулу для вычисления мощности двусторонней тени идеала $\mathcal{M}(n, k)$. Положим

$$
\begin{aligned}
s_{l}(A) & =\min \{i \in[n] \mid i \notin A\}-1, \\
s_{u}(A) & =n-a_{k}, \\
s(A) & =s_{l}(A)+s_{u}(A) .
\end{aligned}
$$

Предложение 5. Пусть $\mathscr{F} \subseteq\left(\begin{array}{c}{[n]} \\ k\end{array}\right)-$ идеал $\mathcal{M}(n, k)$. Тогда

$$
|\nabla \mathscr{F}|=\sum_{A \in \mathscr{F}} s(A) .
$$

Доказательство. Пусть $A$ - максимальная точка идеала $\mathscr{F}$, то есть не существует такого $B \in \mathscr{F}$, что $B \sqsupseteq A$. Обозначим $\mathscr{F}^{\prime}=\mathscr{F} \backslash\{A\}$. Докажем, что

$$
\left|\nabla \mathscr{F}^{\prime}\right|+s(A)=|\nabla \mathscr{F}| \text {. }
$$

1. Покажем, что

$$
\left|\triangle \mathscr{F}^{\prime}\right|+s_{l}(A)=|\triangle \mathscr{F}| .
$$

Пусть $s_{l}(A)=m$, то есть $\{1,2, \ldots, m\} \subseteq A, m+1 \notin A$. Докажем, что

$$
\triangle A \cap \Delta \mathscr{F}^{\prime}=\left\{A \backslash\left\{a_{i}\right\} \mid i>m\right\} .
$$

По определению,

$$
\triangle A=\left\{A \backslash\left\{a_{i}\right\} \mid 1 \leqslant i \leqslant k\right\} .
$$

Если $i>m$, то

$$
B=A \backslash\left\{a_{i}\right\} \cup\{m+1\} \sqsubseteq A,
$$

и $B \neq A$, поэтому $B \in \mathscr{F}^{\prime}$, и $A \backslash\left\{a_{i}\right\}=B \backslash\{m+1\} \in \Delta \mathscr{F}^{\prime}$.

Если $1 \leqslant i \leqslant m$, то

$$
A \backslash\left\{a_{i}\right\}=A \backslash\{i\} .
$$

Предположим, что $A \backslash\{i\} \in \Delta \mathscr{F}^{\prime}$. Тогда $A \backslash\{i\} \cup\{j\} \in \mathscr{F}^{\prime}$. Ясно, что $j \neq i$, так как $A \notin \mathscr{F}^{\prime}$, и $j \geqslant i$, так как $\{1,2, \ldots, i-1\} \subseteq A$. Но в таком случае $A \sqsubseteq A \backslash\{i\} \cup\{j\} \in \mathscr{F}$, и $A$ не является максимальной точкой идеала $\mathscr{F}$, противоречие с выбором $A$. Таким образом,

$$
\left|\Delta A \backslash \Delta \mathscr{F}^{\prime}\right|=m \text {. }
$$

2. Покажем, что

$$
\left|\nabla \mathscr{F}^{\prime}\right|+s_{\mathcal{u}}(A)=|\nabla \mathscr{F}|
$$

Пусть $s_{\mathcal{u}}(A)=m$, то есть $a_{k}=n-m$. Докажем, что

$$
\nabla A \cap \nabla \mathscr{F}^{\prime}=\left\{A \cup\{i\} \mid i \notin A, i<a_{k}\right\} .
$$


По определению,

$$
\nabla A=\{A \cup\{i\} \mid i \notin A\} .
$$

Если $i<a_{k}$, то

$$
B=A \cup\{i\} \backslash\left\{a_{k}\right\} \sqsubseteq A,
$$

поэтому $B \in \mathscr{F}^{\prime}$, и

$$
A \cup\{i\}=B \cup\left\{a_{k}\right\} \in \nabla \mathscr{F}^{\prime} .
$$

Предположим, что $i>a_{k}$, и $A \cup\{i\} \in \nabla \mathscr{F}^{\prime}$. Тогда существует $B=A \cup\{i\} \backslash\{j\} \in \mathscr{F}^{\prime}$. Но $j \neq i$, так как $A \notin \mathscr{F}^{\prime}$. Итак, $j \in A$ и $i>a_{k}$, следовательно, $B \sqsupseteq A$, что противоречит выбору $A$. Таким образом, $A \cup\{i\} \notin \nabla \mathscr{F}^{\prime}$ тогда и только тогда, когда $i>a_{k}$, и

$$
\left|\nabla A \backslash \nabla \mathscr{F}^{\prime}\right|=m \text {. }
$$

Итак, на $\left(\begin{array}{c}{[n]} \\ k\end{array}\right)$ введена такая весовая функция, что мощность двусторонней тени идеала $M(n, k)$ равна его весу, то есть сумме весов его точек. Таким образом, задача о поиске минимальных по двусторонней тени идеалов эквивалентна задаче о нахождении идеалов минимального веса в $M(n, k)$.

Следующее утверждение показывает, что весовые функции $s, s_{u}, s_{l}$ монотонны относительно частичного порядка 巨.

Предложение 6. Если $A \sqsubseteq B$, то

$$
s(A) \geqslant s(B), \quad s_{l}(A) \geqslant s_{l}(B), \quad s_{u}(A) \geqslant s_{u}(B) .
$$

Доказательство. Так как $b_{k} \geqslant a_{k}$, то

$$
s_{u}(B)=n-b_{k} \leqslant n-a_{k}=s_{u}(A) .
$$

Пусть $s_{l}(B)=m$, то есть $b_{1}=1, b_{2}=2, \ldots, b_{m}=m, b_{m+1}>m+1$. Тогда, по определению отношения $\sqsubseteq, a_{1}=1, a_{2}=2, \ldots, a_{m}=m$, и $s_{l}(A) \geqslant m$. Таким образом,

$$
s(A)=s_{l}(A)+s_{u}(A) \geqslant s_{l}(B)+s_{u}(B)=s(B) .
$$

\section{5. Оптимальность $\mathscr{C}_{1}(n, k)$}

Заметим, что семейство $\mathscr{C}_{1}(n, k) \backslash\{\{1,2, \ldots, k\}\}$, как частично упорядоченное множество, изоморфно декартову произведению двух цепей длины $k$ и $n-k$, причем функция $s$ принимает одинаковое значение во всех точках слоя этого ЧУМ.

Пусть $\mathscr{F} \subseteq\left(\begin{array}{c}{[n]} \\ k\end{array}\right)$. Обозначим через $\left.\mathscr{F}\right|_{m}$ совокупность элементов $\mathscr{F}$, в которых функция $s$ принимает значение $m$. Нетрудно видеть, что

$$
\left|\mathscr{C}_{1}(n, k)\right|_{m} \mid= \begin{cases}m+1, & 0 \leqslant m \leqslant \min \{k-1, n-k-1\}, \\ \min \{k, n-k\}, & \min \{k-1, n-k-1\} \leqslant m \leqslant \max \{k-1, n-k-1\}, \\ n-1-m, & \max \{k-1, n-k-1\} \leqslant m \leqslant n-1, \\ 1, & m=n .\end{cases}
$$

На рис. 3 точки $\left(\begin{array}{c}{[5]} \\ 2\end{array}\right)$ и $\left(\begin{array}{c}{[6]} \\ 3\end{array}\right)$ помечены значениями функции $s$. 


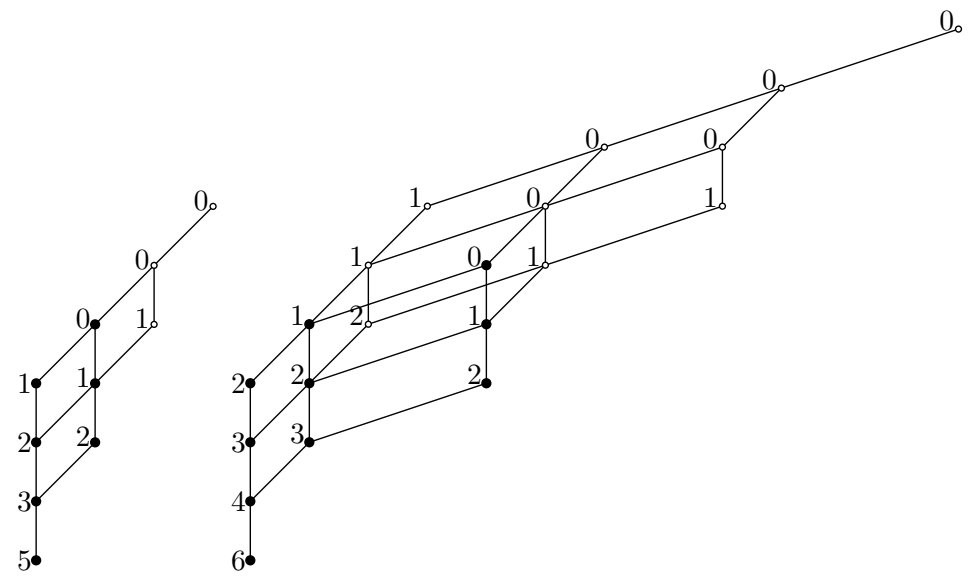

Рис. 3.

Предложение 7. Пусть $A \in\left(\begin{array}{c}{[n]} \\ k\end{array}\right)$ u $s(A)=m$. Тогда существует единственная точка $B \in \mathscr{C}_{1}(n, k)$ такая, что $s(B)=m u B \sqsubseteq A$.

Доказательство. Пусть $s_{l}(A)=m_{1}$ и $s_{u}(A)=m_{2}$, то есть $a_{m_{1}}=m_{1}, a_{m_{1}+1}>m_{1}+1$ и $a_{k}=n-m_{2}$. Рассмотрим точку

$$
B=\left\{1,2, \ldots, m_{1}, m_{1}+2, m_{1}+3, \ldots, k, n-m_{2}\right\} .
$$

Ясно, что $B \in \mathscr{C}_{1}(n, k), B \sqsubseteq A$ и $s(B)=m$. Докажем, что нет других точек, обладающих этими свойствами. Пусть $C \in \mathscr{C}_{1}(n, k), C \sqsubseteq A$ и $s(C)=m$. Тогда из предложения 6 следует, что $s_{l}(C)=m_{1}, s_{u}(C)=m_{2}$, то есть $\left\{1,2, \ldots, m_{1}\right\} \subseteq C, m_{1}+1 \notin C$ и $n-m_{2} \in C$. Из того, что $C \in \mathscr{C}_{1}(n, k)$ следует, что $C \cap\{1,2, \ldots, k\} \geqslant k-1$, значит, $\left\{m_{1}+2, m_{1}+3, \ldots, k\right\} \subseteq C$, то есть $B=C$.

Из предыдущего предложения следует, что семейство точек $\left.\left(\begin{array}{c}{[n]} \\ k\end{array}\right)\right|_{m}$ разбивается на непересекающиеся классы точек, лежащие над точками из $\left.\mathscr{C}_{1}(n, k)\right|_{m}$.

Будем говорить, что $\left.A \in\left(\begin{array}{c}{[n]} \\ k\end{array}\right)\right|_{m}$ порождает $\left.B \in\left(\begin{array}{c}{\left[\begin{array}{c}n \\ k\end{array}\right)} \\ k\end{array}\right)\right|_{m+1}$, если $B \sqsubseteq A$ и не существует такой точки $C$, отличной от $A$ и $B$, что $B \sqsubseteq C \sqsubseteq A$.

Точка $A$ порождает не более двух точек:

(1) если $a_{k-1} \neq a_{k}-1$, то $A$ порождает

$$
B=A \cup\left\{a_{k}-1\right\} \backslash\left\{a_{k}\right\}
$$

(порождение по старшей координате);

(2) если $a_{l}=l, a_{l+1}=l+2$, то $A$ порождает

$$
B=A \cup\{l+1\} \backslash\{l+2\}
$$

(порождение по младшей координате).

Заметим также, что точка $\left.B \in\left(\begin{array}{c}{[n]} \\ k\end{array}\right)\right|_{m+1}$ может порождаться одной из двух точек: 
(1) если $b_{k} \neq n$, то

$$
A=B \cup\left\{b_{k}+1\right\} \backslash\left\{b_{k}\right\}
$$

порождает $B$ по старшей координате;

(2) если $b_{1}=1, b_{2}=2, \ldots, b_{l}=l, b_{l+1}>l+1$, то

$$
A=B \cup\{l+1\} \backslash\{l\}
$$

порождает $B$ по младшей координате.

Наконец, заметим, что точки из $\mathfrak{b}_{1}(n, k)$ порождают только точки из $\mathfrak{b}_{1}(n, k)$ и порождаются только точками из $\mathscr{C}_{1}(n, k)$.

Покажем, что мощность $\left.\mathscr{F}\right|_{m+1}$ не может быть существенно меньше мощности $\left.\mathscr{F}\right|_{m}$. Сначала докажем вспомогательное утверждение.

Предложение 8. Пусть $\mathscr{F} \subseteq\left(\begin{array}{c}{[n]} \\ k\end{array}\right)-$ идеал $\mathcal{M}(n, k)$,

$$
\left|\mathscr{F}_{m}\right| \leqslant\left|\mathscr{C}_{1}(n, k)\right|_{m} \mid, \quad \mathscr{F}^{\prime}=\mathscr{F} \backslash \mathscr{C}_{1}(n, k) .
$$

Тогда выполнено хотя бы одно из условий:

(1) $\left.\left|\mathscr{F}^{\prime}\right|_{m+1}|\geqslant| \mathscr{F}^{\prime}\right|_{m} \mid$;

(2) $\left|\mathscr{F}_{m}\right| \geqslant\left.\left|\mathscr{C}_{1}(n, k)\right|_{m}|-1 u| \mathscr{F}_{m+1}|\geqslant| \mathscr{C}_{1}(n, k)\right|_{m+1} \mid$.

Доказательство. Построим инъективное отображение из $\left.\mathscr{F}^{\prime}\right|_{m}$ в $\left.\mathscr{F}^{\prime}\right|_{m+1}$. Будем рассматривать точки $\left.\mathscr{F}^{\prime}\right|_{m}$ по классам: сначала рассмотрим точки $\left.\mathscr{F}^{\prime}\right|_{m}$, лежащие над лексикографически наибольшей точкой $\left.\mathfrak{b}_{1}(n, k)\right|_{m}$, затем - над второй по порядку точкой, и так далее. Рассматриваемой точке будем ставить в соответствие порождаемую ей точку, причем если текущая точка порождает две еще не задействованные на предыдущих шагах точки, то поставим ей в соответствие точку, порожденную по младшей координате. Рассмотрим случаи, в которых не удается построить такое отображение, то есть на одном из шагов рассматриваемая точка не порождает точек, которые не выбраны на предыдущих шагах.

Заметим, что если $\left.A \in \mathscr{F}\right|_{m}$ порождает точку $\left.B \in \mathscr{F}\right|_{m+1}$ по старшей координате, то к моменту рассмотрения точки $A$ точка $B$ выбрана не будет, так как если $B$ порождается по младшей координате точкой $C$, то эта точка $C$ лежит в следующем классе $\left.\left(\begin{array}{c}{[n]} \\ k\end{array}\right)\right|_{m}$ по отношению к классу точки $A$. Таким образом, шаг процедуры построения инъективного отображения может оказаться неудачным в двух случаях: текущая точка не порождает ни одной точки, или текущая точка порождает одну точку по младшей координате, и эта порожденная точка уже выбрана на одном из предыдущих шагов.

1. Пусть $\left.\mathscr{F}^{\prime}\right|_{m}$ содержит точку $A$, у которой вообще нет порожденных точек в $\left.\mathscr{F}^{\prime}\right|_{m+1}$, и пусть $s_{l}(A)=p, s_{u}(A)=m-p$. Тогда

$$
B=\{1,2, \ldots, p, p+3, p+4, \ldots, k, n-m+p-1, n-m+p\} \sqsubseteq A,
$$

поэтому $B \in \mathscr{F}$. Точки $A$ и $B$ лежат над точкой

$$
C=\left.\{1,2, \ldots, p, p+2, p+3, \ldots, k, n-m+p\} \in \mathscr{C}_{1}(n, k)\right|_{m},
$$


и семейство $\left.\mathscr{F}\right|_{m}$ содержит сегмент $[C, B]$. При $p<k-2$ и $n-m+p>k+2$ выполнено $m<n-5$, и число точек в сегменте равно

$$
(n-m+p-1-k)(k-p-1) \geqslant 2(n-m-3)>\left|\mathscr{C}_{1}(n, k)\right|_{m} \mid,
$$

что противоречит условию утверждения. Если $p=k-2$, то длина цепи $[C, B]$ равна $n-m+k-3-(k-1)=n-m-2$. Следовательно,

$$
\left|\mathscr{F}_{m}\right| \geqslant n-m-2 \text {. }
$$

Если $m<\max \{k-2, n-k-2\}$, то

$$
\left|\mathscr{F}_{m}\right| \geqslant n-m-1>\left|\mathscr{C}_{1}(n, k)\right|_{m} \mid,
$$

что противоречит условию.

Если $m \geqslant \max \{k-2, n-k-2\}$, то

$$
\left|\mathscr{F}_{\mid}\right| \geqslant n-m-2 \geqslant\left|\mathscr{C}_{1}(n, k)\right|_{m} \mid-1 .
$$

Так как точка $C$ порождает две точки в $\left.\mathscr{C}_{1}(n, k)\right|_{m+1}$ и каждая точка $[C, B]$, кроме $B$, порождает точку вне $\left.\mathscr{C}_{1}(n, k)\right|_{m+1}$, верно, что

$$
\left|\mathscr{F}_{m+1}\right| \geqslant n-m-2 \geqslant\left|\mathscr{C}_{1}(n, k)\right|_{m+1} \mid \text {. }
$$

2. Пусть $\left.\mathscr{F}^{\prime}\right|_{m}$ содержит точку $A$, которая порождает одну точку $\left.B \in \mathscr{F}^{\prime}\right|_{m+1}$ по младшей координате, и точка $B$ уже выбрана при рассмотрении предыдущего класса.

Пусть

$$
s_{l}(A)=p, \quad s_{u}(A)=m-p .
$$

Поскольку $A$ порождает точку по младшей координате, верно, что $\{1,2, \ldots, p, p+2\} \subseteq A$. Так как $A$ не порождает точку по старшей координате, $\{n-m+p-1, n-m+p\} \subseteq A$. Поскольку $A \notin \mathscr{C}_{1}(n, k)$, верно, что $n-m+p \geqslant k+2$ и $p \leqslant k-3$, поэтому

$$
m \leqslant n-5 \text {. }
$$

Пусть $\{p+2, p+3, \ldots, p+q\} \subseteq A, p+q+1 \notin A$. Заметим, что $q \geqslant 2$. Таким образом,

$$
\begin{aligned}
A=\{1,2, \ldots, p, p+2, p+3, \ldots, p+q, & \\
& \left.a_{p+q}, a_{p+q+1}, \ldots, a_{k-2}, n-m+p-1, n-m+p\right\} .
\end{aligned}
$$

Порожденная $A$ точка имеет вид

$$
\begin{aligned}
\hat{A}=\{ & 1,2, \ldots, p+1, p+3, p+4, \ldots, p+q, \\
& \left.a_{p+q}, a_{p+q+1}, \ldots, a_{k-2}, n-m+p-1, n-m+p\right\} .
\end{aligned}
$$

Эта точка уже выбрана, значит,

$$
\begin{aligned}
B=\{ & 1,2, \ldots, p+1, p+3, p+4, \ldots, p+q, \\
& \left.a_{p+q}, a_{p+q+1}, \ldots, a_{k-2}, n-m+p-1, n-m+p+1\right\} \in \mathscr{F} .
\end{aligned}
$$


Если $q>2$, то $B$ порождает по младшей координате точку $\widehat{B}$, и, так как точке $B$ была поставлена в соответствие точка $\hat{A}$, то

$$
\begin{aligned}
\widehat{B}=\{ & 1,2, \ldots, p+2, p+4, p+5, \ldots, p+q, \\
& \left.a_{p+q}, a_{p+q+1}, \ldots, a_{k-2}, n-m+p-1, n-m+p+1\right\}
\end{aligned}
$$

уже была выбрана, то есть

$$
\begin{aligned}
& C=\{1,2, \ldots, p+2, p+4, p+5, \ldots, p+q, \\
&\left.a_{p+q}, a_{p+q+1}, \ldots, a_{k-2}, n-m+p-1, n-m+p+2\right\} \in \mathscr{F} .
\end{aligned}
$$

Продолжая, получим, что

$$
\begin{gathered}
\left\{1,2, \ldots, p, p+2, p+3, \ldots, p+q, a_{p+q}, a_{p+q+1}, \ldots, a_{k-2},\right. \\
n-m+p-1, n-m+p\} \in \mathscr{F}, \\
\left\{1,2, \ldots, p+1, p+3, p+4, \ldots, p+q, a_{p+q}, a_{p+q+1}, \ldots, a_{k-2},\right. \\
n-m+p-1, n-m+p+1\} \in \mathscr{F}, \\
\ldots \\
\left\{1,2, \ldots, p+q-1, a_{p+q}, a_{p+q+1}, \ldots, a_{k-2},\right. \\
n-m+p-1, n-m+p+q-1\} \in \mathscr{F},
\end{gathered}
$$

и в случае $m<n-5$

$$
\begin{aligned}
\left|\mathscr{F}_{\mid}\right| & \geqslant q(n+m+p-1-p-q)=q(n-m-q-1) \\
& \geqslant 2(n-m-3)>\left|\mathscr{C}_{1}(n, k)\right|_{m} \mid .
\end{aligned}
$$

В случае $m=n-5$ получаем

$$
\begin{aligned}
|\mathscr{F}|_{m} \mid & \geqslant 4=\left|\mathscr{C}_{1}(n, k)\right|_{m} \mid, \\
|\mathscr{F}|_{m+1} \mid \geqslant 3 & =\left|\mathscr{C}_{1}(n, k)\right|_{m+1} \mid .
\end{aligned}
$$

Таким образом, если условия утверждения выполнены, то либо можно построить инъективное отображение из $\left.\mathscr{F}^{\prime}\right|_{m}$ в $\left.\mathscr{F}^{\prime}\right|_{m+1}$, то есть

$$
\left.\left|\mathscr{F}^{\prime}\right|_{m+1}|\geqslant| \mathscr{F}^{\prime}\right|_{m} \mid
$$

либо

$$
\left.|\mathscr{F}|_{m}|\geqslant| \mathscr{C}_{1}(n, k)\right|_{m} \mid-1
$$

и

$$
\left|\mathscr{F}_{m+1}\right| \geqslant\left|\mathscr{C}_{1}(n, k)\right|_{m+1} \mid
$$

Теперь получим из доказанного утверждения два следствия о зависимости мощности $\left.\mathscr{F}\right|_{m+1}$ от мощности $\left.\mathscr{F}\right|_{m}$. 
Предложение 9. Пусть Ғㅍ - идеал $\mathcal{M}(n, k)$. Тогда если

$$
\left|\mathscr{F}_{m}\right| \geqslant\left|\mathscr{C}_{1}(n, k)\right|_{m} \mid,
$$

mo

$$
\left.|\mathscr{F}|_{m+1}|\geqslant| \mathscr{C}_{1}(n, k)\right|_{m+1} \mid .
$$

Доказательство. Пусть $\mathscr{F}^{\prime} \subseteq \mathscr{F}-$ идеал, и

$$
\left.\left|\mathscr{F}^{\prime}\right|_{m}|=| \mathscr{C}_{1}(n, k)\right|_{m} \mid \text {. }
$$

Тогда по предложению 8 либо

$$
\left.\left|\mathscr{F}^{\prime}\right|_{m+1}|\geqslant| \mathscr{C}_{1}(n, k)\right|_{m+1} \mid,
$$

то есть выполнено утверждение, либо

$$
\left.\left|\left(\mathscr{F}^{\prime} \backslash \mathscr{C}_{1}(n, k)\right)\right|_{m+1}|\geqslant|\left(\mathscr{F}^{\prime} \backslash \mathscr{C}_{1}(n, k)\right)\right|_{m} \mid .
$$

Остается показать, что во втором случае выполнено также

$$
\left.\left.\left.\left|\left(\mathscr{F}^{\prime} \cap \mathscr{C}_{1}(n, k)\right)\right|_{m+1}|\geqslant|\left(\mathscr{F}^{\prime} \cap \mathscr{C}_{1}(n, k)\right)\right|_{m}|+| \mathscr{C}_{1}(n, k)\right|_{m+1}|-| \mathscr{C}_{1}(n, k)\right|_{m} \mid,
$$

но это следует из того, что $\mathscr{C}_{1}(n, k) \backslash\{\{1,2, \ldots, k\}\}$ изоморфно, как ЧУМ, декартову произведению цепей, причем $\left.\mathscr{C}_{1}(n, k)\right|_{m}$ соответствует при изоморфизме слою этого произведения, и из теоремы Клементса-Линдстрема.

Предложение 10. Пусть Ғ - идеал $\mathcal{M}(n, k)$. Тогда, если $\left.|\mathscr{F}|_{m}|<| \mathscr{C}_{1}(n, k)\right|_{m} \mid$, то выполнено хотя бы одно из условий:

(1) $\left|\mathscr{F}_{m+1}\right| \geqslant\left|\mathscr{F}_{m}\right|+\max \left\{\left.\left|\mathscr{C}_{1}(n, k)\right|_{m+1}|-| \mathscr{C}_{1}(n, k)\right|_{m} \mid, 0\right\}$,

(2) $\left.|\mathscr{F}|_{m+1}|\geqslant| \mathscr{C}_{1}(n, k)\right|_{m+1} \mid$.

Доказательство. Если выполнено второе условие предложения 8, то выполнено второе условие данного предложения.

Если выполнено первое условие предложения 8, то доказываемое предложение вытекает из того, что

$$
\left.\left|\left(\mathscr{F} \backslash \mathscr{C}_{1}(n, k)\right)\right|_{m+1}|\geqslant|\left(\mathscr{F} \backslash \mathscr{C}_{1}(n, k)\right)\right|_{m} \mid
$$

по предложению 8, а

$$
\left.\left|\left(\mathscr{F} \cap \mathscr{C}_{1}(n, k)\right)\right|_{m+1}|\geqslant|\left(\mathscr{F} \cap \mathscr{C}_{1}(n, k)\right)\right|_{m} \mid+\max \left\{\left.\left|\mathscr{C}_{1}(n, k)\right|_{m+1}|-| \mathscr{C}_{1}(n, k)\right|_{m} \mid, 0\right\}
$$

по теореме Клементса-Линдстрема.

Теорема 1. Верно, что $\mathscr{C}_{1}(n, k)-$ минимальное по двусторонней тени семейство, то есть если $\mathscr{F} \subseteq\left(\begin{array}{c}{[n]} \\ k\end{array}\right)$ u $|\mathscr{F}|=\left|\mathscr{C}_{1}(n, k)\right|$, то $|\nabla \mathscr{F}| \geqslant\left|\nabla \mathscr{C}_{1}(n, k)\right|$. Более того, если $\mathscr{F}-$ идеал $u|\nabla \mathscr{F}|=\left|\nabla \mathscr{C}_{1}(n, k)\right|$, mo $\mathscr{F}=\mathscr{C}_{1}(n, k)$. 
Доказательство. Предположим противное, пусть $|\mathscr{F}|=\left|\mathscr{C}_{1}(n, k)\right|$ и $|\nabla \mathscr{F}|<\left|\nabla \mathscr{C}_{1}(n, k)\right|$. Так как сдвиг не увеличивает двустороннюю тень, можно предположить, не ограничивая общности, что $\mathscr{F}$ - идеал $\mathcal{M}(n, k)$. Тогда

$$
|\nabla \mathscr{F}|=\sum_{A \in \mathscr{F}} s(A)=\left.\sum_{m=0}^{n} m|\mathscr{F}|_{m}\left|<\sum_{m=0}^{n} m\right| \mathscr{C}_{1}(n, k)\right|_{m}|=| \nabla \mathscr{C}_{1}(n, k) \mid .
$$

Из этого неравенства следует, что найдется такое $m_{1}$, что

$$
\left|\mathscr{F}_{m_{1}}\right|<\left|\mathscr{C}_{1}(n, k)\right|_{m_{1}} \mid \text {. }
$$

Выберем в качестве $m_{1}$ наибольшее из таких чисел. Так как $|\mathscr{F}|=\left|\mathscr{C}_{1}(n, k)\right|$, найдется такое $m_{2}$, что

$$
\left|\mathscr{F}_{m_{2}}\right|>\left|\mathscr{C}_{1}(n, k)\right|_{m_{2}} \mid
$$

причем можно выбрать $m_{2}$ так, что $m_{2}<m_{1}$, потому что в противном случае

$$
\sum_{m=0}^{n} m\left|\mathscr{F}_{m}\right|>\sum_{m=0}^{n} m\left|\mathscr{C}_{1}(n, k)\right|_{m} \mid
$$

Но из предложения 9 следует, что если $\left.\left|\mathscr{F}_{\mid}\right|_{m_{2}}|>| \mathscr{C}_{1}(n, k)\right|_{m_{2}} \mid$, то $\left|\mathscr{F}_{m_{1}}\right| \geqslant\left|\mathscr{C}_{1}(n, k)\right|_{m_{1}} \mid$. Получаем противоречие с выбором $m_{1}$. Таким образом, для любого идеала $\mathscr{F}$, мощность которого равна $\left|\mathscr{C}_{1}(n, k)\right|$, верно, что

$$
|\nabla \mathscr{F}| \geqslant\left|\nabla \mathscr{C}_{1}(n, k)\right| \text {. }
$$

Докажем единственность минимального идеала. Пусть $\mathscr{F}-$ идеал, $|\mathscr{F}|=\left|\mathscr{C}_{1}(n, k)\right|$, и $|\nabla \mathscr{F}|=\left|\nabla \mathscr{C}_{1}(n, k)\right|$. Тогда

$$
\left.\sum_{m=0}^{n} m|\mathscr{F}|_{m}\left|=\sum_{m=0}^{n} m\right| \mathscr{C}_{1}(n, k)\right|_{m} \mid
$$

Поскольку предположение о том, что найдется $m_{1}$ такое, что $\left|\mathscr{F}_{m_{1}}\right|<\left|\mathscr{C}_{1}(n, k)\right|_{m_{1}} \mid$, приводит к противоречию, верно, что

$$
\left|\mathscr{F}_{m}\right|=\left|\mathscr{C}_{1}(n, k)\right|_{m} \mid
$$

при всех $m$. Итак,

$$
\left|\mathscr{F}_{0}\right|=\left|\mathscr{C}_{1}(n, k)\right|_{0} \mid=1,
$$

поэтому наименьшая точка $\{2,3, \ldots, k, n\}$ семейства $\left.\left(\begin{array}{c}{[n]} \\ k\end{array}\right)\right|_{0}$ принадлежит $\mathscr{F}$. Поскольку $\{2,3, \ldots, k, n\}-$ наибольшая точка идеала $\mathscr{C}_{1}(n, k)$, верно, что $\mathscr{C}_{1}(n, k) \subseteq \mathscr{F}$, и следовательно, $\mathscr{C}_{1}(n, k)=\mathscr{F}$.

Теперь, используя свойства оператора сдвига, докажем, что все минимальные семейства мощности $\left|\mathscr{C}_{1}(n, k)\right|$, не являющиеся идеалами $\mathcal{M}(n, k)$, изоморфны $\mathscr{C}_{1}(n, k)$.

Теорема 2. Верно, что $\mathscr{b}_{1}(n, k)$ - единственное с точностью до изоморфизма (перестановки координат) минимальное по двусторонней тени семейство мощности $1+k(n-k)$. 
Доказательство. Сначала докажем, что $S_{i j}\left(\mathscr{C}_{1}(n, k)\right)$ совпадает с семейством, соответствующим кругу $\mathscr{C}_{1}(\tilde{\alpha})$ для некоторого $\tilde{\alpha} \in B_{k}^{n}$.

Если $i \leqslant k, j \leqslant k$ или $i>k, j>k$, то, поскольку $\{i\} \cup B \in \mathscr{C}_{1}(n, k)$ тогда и только тогда, когда $\{j\} \cup B \in \mathscr{C}_{1}(n, k)$, верно, что

$$
S_{i j}\left(\mathscr{C}_{1}(n, k), A\right)=A
$$

для любого $A \in \mathscr{C}_{1}(n, k)$, и поэтому

$$
S_{i j}\left(\mathscr{C}_{1}(n, k)\right)=\mathscr{C}_{1}(n, k) .
$$

Если $i \leqslant k, j>k$, то, поскольку из того, что $\{j\} \cup B \in \mathscr{C}_{1}(n, k)$, следует, что $\{i\} \cup B \in \mathscr{C}_{1}(n, k)$, также выполнено

$$
S_{i j}\left(\mathscr{C}_{1}(n, k), A\right)=A
$$

для любого $A \in \mathscr{C}_{1}(n, k)$, и

$$
S_{i j}\left(\mathscr{C}_{1}(n, k)\right)=\mathscr{C}_{1}(n, k) .
$$

Пусть $i>k, j \leqslant k$. Покажем, что $S_{i j}\left(\mathscr{C}_{1}(n, k)\right)$ совпадает с семейством $\mathscr{F}$, соответствующим кругу $\mathscr{C}_{1}(0 \ldots 01^{i} 0 \ldots 0 \underbrace{1 \ldots 101 \ldots 1}_{k})$. Пусть $A \in \mathscr{F}$, докажем, что $A \in S_{i j}\left(\mathscr{C}_{1}(n, k)\right)$. Если $|A \cap\{1,2, \ldots, k\}| \geqslant k-1$, то $A \in \mathscr{C}_{1}(n, k)$, и $S_{i j}\left(\mathscr{C}_{1}(n, k), A\right)=A$, так как либо $j \notin A$, либо $A=\{1,2, \ldots, k\}$. В противном случае

$$
A=\{1,2, \ldots, j-1, j+1, j+2, \ldots, k, i, s\} \backslash\{t\},
$$

где $s>k, t \leqslant k$, и

$$
A=S_{i j}\left(\mathscr{C}_{1}(n, k), A \cup\{j\} \backslash\{i\}\right) .
$$

Поскольку

$$
\left|S_{i j}\left(\mathscr{C}_{1}(n, k)\right)\right|=\left|\mathscr{C}_{1}(n, k)\right|,
$$

верно, что

$$
S_{i j}\left(\mathscr{C}_{1}(n, k)\right)=\mathscr{F} .
$$

Теперь предположим, что существует минимальное по двусторонней тени семейство $\mathscr{F},|\mathscr{F}|=1+k(n-k)$, и $\mathscr{F}$ не изоморфно $\mathscr{C}_{1}(n, k)$. Так как $\mathscr{C}_{1}(n, k)-$ единственный минимальный по двусторонней тени идеал $\mathcal{M}(n, k)$ мощности $|\mathscr{F}|$, существует последо-

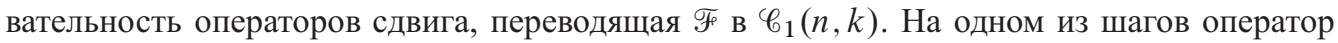
сдвига переводит семейство, не изоморфное $\mathscr{b}_{1}(n, k)$, в семейство, изоморфное $\mathscr{b}_{1}(n, k)$, поэтому существует такое минимальное по двусторонней тени семейство $\mathscr{F}^{\prime}$, не изоморфное $\mathscr{C}_{1}(n, k)$, что $S_{i j}\left(\mathscr{F}^{\prime}\right)$ изоморфно $\mathscr{C}_{1}(n, k)$. Не ограничивая общности, можно считать, что $S_{i j}\left(\mathscr{F}^{\prime}\right)=\mathscr{C}_{1}(n, k)$. Так как $\mathscr{F}^{\prime} \neq \mathscr{C}_{1}(n, k)$, существует $A$ такое, что $\{i\} \cup A \in \mathscr{C}_{1}(n, k)$, a $\{j\} \cup A \in \mathscr{F}^{\prime} \backslash \mathscr{C}_{1}(n, k)$. Из этого следует, что $i \leqslant k, j>k$. Таким образом, $\mathscr{F}^{\prime} \backslash \mathscr{C}_{1}(n, k)$ состоит из элементов вида $\{j\} \cup A$, где $A$ таково, что $\{i\} \cup A \in \mathscr{C}_{1}(n, k)$, то есть

$$
|A \cap\{1,2, \ldots, k\}|=k-2 .
$$


Если $\{j\} \cup A \in \mathscr{F}^{\prime}$ для всех таких $A$, то $\mathscr{F}^{\prime}=S_{j i}\left(\mathscr{C}_{1}(n, k)\right)$ и изоморфно $\mathscr{C}_{1}(n, k)$, что противоречит выбору $\mathscr{F}^{\prime}$. Поэтому существуют $A_{1}, A_{2}$ такие, что $\{i\} \cup A_{1} \in \mathscr{F}^{\prime}$, $\{j\} \cup A_{2} \in \mathscr{F}^{\prime}$,

$$
\begin{aligned}
& \left|A_{1} \cap(\{1,2, \ldots, k\} \backslash\{i, j\})\right|=k-2, \\
& \left|A_{2} \cap(\{1,2, \ldots, k\} \backslash\{i, j\})\right|=k-2 .
\end{aligned}
$$

Но поскольку для любого такого $A$, что $|A \cap(\{1,2, \ldots, k\} \backslash\{i, j\})|=k-2$ ровно один из элементов $\{i\} \cup A,\{j\} \cup A$ входит в $\mathscr{F}^{\prime}$, то можно выбрать $A_{1}$ и $A_{2}$ так, что

$$
\left|A_{1} \cap A_{2}\right|=k-2 \text {. }
$$

Заметим, что $A_{1} \backslash A_{2}=\{m\}$, и в случае $m \in\{1,2, \ldots, k\}$ выполнено

$$
\Delta\left(\{j\} \cup A_{2}\right) \nsubseteq \Delta S_{i j}\left(\mathscr{F}^{\prime}\right)=\Delta \mathscr{C}_{1}(n, k),
$$

а в случае $m \in\{k+1, k+2, \ldots, n\}$ выполнено

$$
\nabla\left(\{j\} \cup A_{2}\right) \nsubseteq \nabla \mathscr{C}_{1}(n, k),
$$

и по предложению 2

$$
\left|\nabla_{\triangle} \mathscr{C}_{1}(n, k)\right|=\left|{ }_{\triangle} S_{i j}\left(\mathscr{F}^{\prime}\right)\right|<\left|\nabla_{\triangle} \mathscr{F}^{\prime}\right|,
$$

что противоречит минимальности $\mathscr{F}^{\prime}$. Таким образом, предположение о существовании минимального по двусторонней тени $\mathscr{F}$, не изоморфного $\mathscr{C}_{1}(n, k)$, приводит к противоречию.

Следующее утверждение показывает, что для задачи с двусторонней тенью нельзя доказать аналог теоремы Краскала - Катоны, то есть задача минимизации двусторонней тени существенно отличается от задачи минимизации тени вниз.

Предложение 11. Если $n \geqslant 8$, то на $\left(\begin{array}{c}{[n]} \\ 3\end{array}\right)$ нельзя ввести линейный порядок так, чтобы все его начальные отрезки являлись минимальными по двусторонней тени семействами.

Доказательство. Предположим, на $\left(\begin{array}{c}{[n]} \\ 3\end{array}\right)$ существует линейный порядок с требуемым свойством. Тогда, поскольку $\mathscr{C}_{1}(n, 3)-$ единственное с точностью до изоморфизма минимальное семейство мощности $3 n-8$, начальный отрезок этой длины должен совпадать с $\mathscr{C}_{1}(n, 3)$.

Рассмотрим начальный отрезок длины $4 n-14$, обозначим его через $\mathscr{L}$. Пусть $S(\mathscr{L})-$ идеал $\mathscr{b}(n, 3)$, полученный из $\mathscr{L}$ применением оператора сдвига, тогда по свойству линейного порядка и оператора сдвига $S(\mathscr{L})$ - минимальное по двусторонней тени семейство. Заметим, что $\mathscr{C}_{1}(n, 3) \subseteq S(\mathscr{L})$. Оценим снизу мощность $\triangle S(\mathscr{L})$. Из того, что

$$
\left|S(\mathscr{L}) \backslash \mathscr{C}_{1}(n, 3)\right|=n-6,
$$

и из предложения 9 следует, что

$$
\sum_{A \in S(\mathscr{L}) \backslash \mathfrak{b}_{1}(n, 3)} S(A) \geqslant n-4+n-5+\ldots+3=\frac{(n-1)(n-6)}{2} .
$$

Таким образом,

$$
|\nabla S(\mathscr{L})| \geqslant\left|\nabla \mathscr{C}_{1}(n, 3)\right|+\frac{(n-1)(n-6)}{2} .
$$

5 Дискретная математика, т.23 №4 
Теперь рассмотрим семейство

$$
\mathscr{F}=\{A \mid 1 \in A, A \cap\{2,3,4,5\} \neq \varnothing\} .
$$

Очевидно, что

$$
|\mathscr{F}|=n-2+n-3+n-4+n-5=4 n-14
$$

и

$$
|\nabla \mathscr{F}|=\left|\nabla \mathscr{C}_{1}(n, 3)\right|+\frac{(n-4)(n-5)}{2}<|\nabla S(\mathscr{L})|,
$$

что противоречит минимальности $S(\mathscr{L})$.

\section{6. Оптимальные семейства малой мощности}

Пусть

$$
0 \leqslant r \leqslant\left|\mathscr{C}_{1}(n, k)\right|=1+k(n-k) .
$$

Обозначим через $\mathscr{L}_{r}$ начальный лексикографический отрезок $\mathscr{C}_{1}(n, k)$ мощности $r$. Заметим, что

$$
\begin{aligned}
\mathscr{L}_{0} & =\varnothing, \\
\mathscr{L}_{r+1} & =\mathscr{L}_{\boldsymbol{r}} \cup\left\{\min _{\mathrm{lex}}\left\{A \in \mathscr{C}_{1}(n, k) \backslash \mathscr{L}_{\boldsymbol{r}}\right\}\right\} .
\end{aligned}
$$

Семейства $\mathscr{L}_{\boldsymbol{r}}$ несдвигаемы, так как $A \sqsubseteq B$ влечет $A \leqslant_{\text {lex }} B$. Также ясно, что семейства $\left.\mathscr{L}_{r}\right|_{m}$ являются начальными лексикографическими отрезками семейства $\left.\mathscr{C}_{1}(n, k)\right|_{m}$. Если $r=1+k p+q, 0 \leqslant q<k$, то

$$
\begin{aligned}
\mathscr{L}_{r}=\{ & \{1,2, \ldots, k\}\} \\
& \cup\{\{1,2, \ldots, k\} \backslash\{m\} \cup\{k+i\} \mid m=1,2, \ldots, k ; i=1,2, \ldots, p\} \\
& \cup\{\{1,2, \ldots, k\} \backslash\{m\} \cup\{k+p+1\} \mid m=k-q+1, k-q+2, \ldots, k\} .
\end{aligned}
$$

На рис. 4 изображено семейство $\mathscr{C}_{1}(8,4)$ и выделены точки семейства $\mathscr{L}_{11}$.

Обозначим через $\mathscr{R}_{r}, 0 \leqslant r \leqslant 1+k(n-k)$ семейства следующего вида:

$$
\begin{aligned}
\mathscr{R}_{0} & =\varnothing, \\
\mathscr{R}_{r+1} & =\mathscr{R}_{r} \cup\left\{\max _{\text {lex }}\left\{A \in \mathscr{C}_{1}(n, k) \backslash \mathscr{R}_{r} \mid \mathscr{R}_{r} \cup\{A\}-\text { идеал }\right\}\right\} .
\end{aligned}
$$

Очевидно, $\mathscr{R}_{r}$ являются несдвигаемыми, и семейства $\left.\mathscr{R}_{r}\right|_{m}$ являются конечными лексикографическими отрезками $\left.\mathscr{C}_{1}(n, k)\right|_{m}$. Если $r=1+(n-k) p+q, 0 \leqslant q<n-k$, To

$$
\begin{aligned}
\mathscr{R}_{r}=\{\{1,2, \ldots, k\}\} \\
\cup\{\{1,2, \ldots, k\} \backslash\{m\} \cup\{i\} \mid m=k-p+1, k-p+2, \ldots, k ; \\
i=k+1, k+2, \ldots, n\} \\
\cup\{\{1,2, \ldots, k\} \backslash\{k-p\} \cup\{i\} \mid i=k+1, k+2, \ldots, k+q\} .
\end{aligned}
$$

На рис. 5 изображено семейство $\mathscr{C}_{1}(8,4)$ и выделены точки семейства $\mathscr{R}_{11}$. 


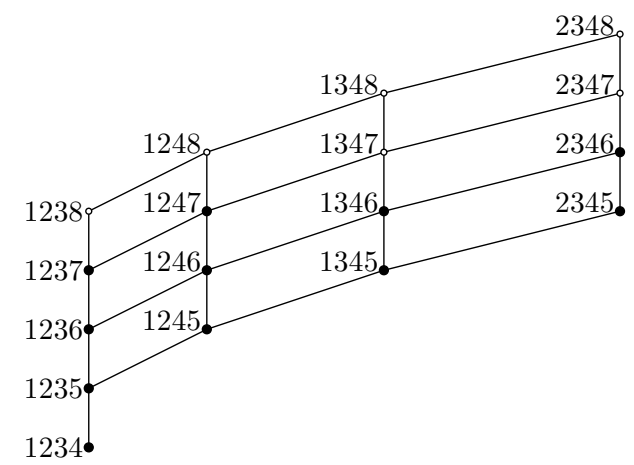

Рис. 4.

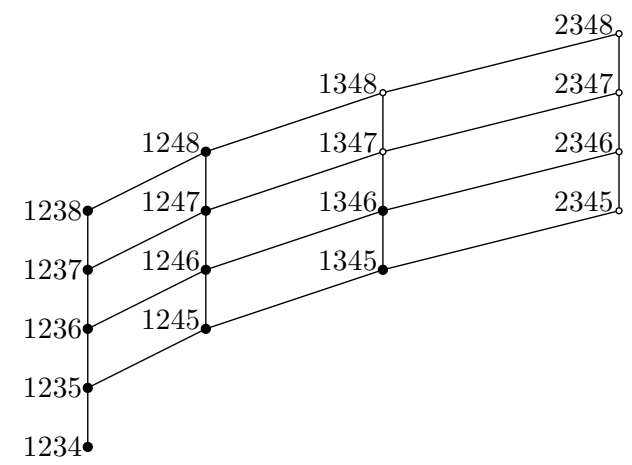

Pис. 5.

Теорема 3. Если $k \leqslant n / 2$, то семейства $\mathscr{R}_{r}$ являются минимальными по двусторонней тени. Если $k \geqslant n / 2$, то семейства $\mathscr{L}_{r}$ являются минимальными по двусторонней тени.

Доказательство. Доказательства для случаев $k \leqslant n / 2$ и $k \geqslant n / 2$ аналогичны; докажем теорему для $k \geqslant n / 2$.

Пусть $\mathscr{F}$ - идеал $M(n, k)$, и $|\mathscr{F}|=r, 0<r<\left|\mathscr{C}_{1}(n, k)\right|$.

Докажем утверждение от противного: предположим, что $|\nabla \mathscr{F}|<\left|\nabla \mathscr{L}_{r}\right|$. По предложению 5 ,

$$
|\nabla \mathscr{F}|=\sum_{A \in \mathscr{F}} s(A)=\left.\sum_{m=0}^{n} m|\mathscr{F}|_{m}\left|<\sum_{m=0}^{n} m\right| \mathscr{L}_{r}\right|_{m}|=| \nabla^{\nabla} \mathscr{L}_{r} \mid,
$$

поэтому существуют $m_{1}$ и $m_{2}$ такие, что $m_{1}>m_{2},\left|\mathscr{F}_{m_{1}}\right|<\left|\mathscr{L}_{r}\right|_{m_{1}} \mid$ и $\left|\mathscr{F}_{m_{2}}\right|>\left|\mathscr{L}_{r}\right|_{m_{2}} \mid$. Заметим, что

$$
\left.|\mathscr{F}|_{m_{2}}|<| \mathscr{C}_{1}(n, k)\right|_{m_{2}} \mid,
$$

иначе по предложению $9\left|\mathscr{F}_{m_{1}}\right| \geqslant\left.\left|\mathscr{C}_{1}(n, k)\right|_{m_{1}}|\geqslant| \mathscr{L}_{r}\right|_{m_{1}} \mid$, что противоречит выбору $m_{1}$. Поэтому к $\left.\mathscr{F}\right|_{m_{2}}$ применимо предложение 10 , и, поскольку второе условие этого предложения приводит к тому же противоречию, верно, что

$$
\left|\mathscr{F}_{m_{2}+1}\right| \geqslant\left|\mathscr{F}_{m_{2}}\right|+\max \left\{\left.\left|\mathscr{C}_{1}(n, k)\right|_{m_{2}+1}|-| \mathscr{C}_{1}(n, k)\right|_{m_{2}} \mid, 0\right\} .
$$


Пусть $r=1+p k+q, 0 \leqslant q<k$. Из определения семейства $\mathscr{L}_{r}$ следует, что

$$
\left.\left|\mathscr{L}_{r}\right|_{m+1}|-| \mathscr{L}_{r}\right|_{m} \mid \leqslant \max \left\{\left.\left|\mathscr{C}_{1}(n, k)\right|_{m+1}|-| \mathscr{C}_{1}(n, k)\right|_{m} \mid, 0\right\}
$$

при всех $m$ за исключением, быть может, одного значения $m$ в случае $q>0$, при котором эта разность составляет $\max \left\{\left.\left|\mathscr{C}_{1}(n, k)\right|_{m+1}|-| \mathscr{C}_{1}(n, k)\right|_{m} \mid, 0\right\}+1$. Отсюда и из (*) следует, что $\left.|\mathscr{F}|_{m_{2}}|>| \mathscr{L}_{\boldsymbol{r}}\right|_{m_{2}} \mid$ влечет $\left.|\mathscr{F}|_{m_{1}}|\geqslant| \mathscr{L}_{\boldsymbol{r}}\right|_{m_{1}} \mid$, что противоречит свойству $m_{1}$.

\section{Список литературы}

1. Сапоженко А. А., Проблема Дедекинда и метод граничных функционалов. Физматлит, Москва, 2009.

2. Kruskal J., The number of simplices in a complex. In: Mathematical Optimization Techniques. Univ. of California Press, Berkeley, 1963, pp. 251-278.

3. Katona G. O. H., A theorem of finite sets. In: Proc. Tihany Conf., 1966, pp. 187-207.

4. Mörs M., A generalization of a theorem of Kruskal. Graphs and Combinatorics (1985) 1, 167-183.

5. Füredi Z., Griggs J. R., Families of finite sets with minimal shadows. Combinatorica (1986) 6, $355-363$.

6. Clements G. F., Lindström B., A generalization of a combinatorial theorem of Macaulay. J. Combinatorial Theory (1969) 7, 230-238.

7. Безруков С. Л., Минимизация теней подмножеств полурешетки частичных отображений. Дискретный анализ и исследование операџий (1988) 47, 3-18.

8. Bezrukov S. L., Leck U., Macaulay posets. Electron. J. Comb. (2004) DS12.

9. Frankl P., Shadows and shifting. Graphs and Combinatorics (1991) 7, 23-29.

10. Ahlswede R., Aydinian H., Khachatrian L. H., More about shifting techniques. European J. Combin. (2003) 24, 551-556.

Статья поступила 17.11.2010. 